\title{
Nontuberculous mycobacterial infections in children in the Czech Republic in the period 2003-2018
}

\author{
Karolina Dolezalova ${ }^{\mathrm{a}, \mathrm{d}}$, Marek Maly $^{\mathrm{b}}$, Jiri Wallenfels ${ }^{c}$, Dana Gopfertova ${ }^{\mathrm{d}}$
}

\begin{abstract}
Aim. Comparision of the incidence of cervical lymphadenitis caused by nontuberculous mycobacteria in two equal time periods before and after the ending of widespread calmetization (tuberculosis vaccination).

Backgroung. From 2011 to 2018, 89 children were registered in the Tuberculosis Register with cervical lymphadenitis caused by nontuberculous mycobacteria, as confirmed by cultivation. In the majority of cases, the infection was caused by a mycobacterium belonging to the Mycobacterium avium complex. Only 7 cases of cervical lymphadenitis of the same etiology were registered during the same time interval between 2003 and 2010. The authors consider the ending of widespread calmetization (tuberculosis vaccination) in 2010 to be the main cause of the growing incidence. Method. A comparison of data for the period 2003-2018 about cases of the illness caused by atypical mycobacteria as reported in the Tuberculosis Register.

Results. The average incidence per year in the first interval was $0.04 / 100000$ children and in the second interval $0.53 / 100000$ children. During the second time interval, there was an increase from $0.14 / 100000$ children in 2011 to $1.40 / 100000$ children in 2018. While the incidence during the first time interval did not show any time trend $(P=0.885)$, the year 2010 marks a significant turning point, with growth during the second interval being highly statistically significant $(P<0.001)$.
\end{abstract}

Key words: nontuberculous mycobacterial infection (NTM), Mycobacterium avium complex, cervical lymphadenitis, calmetization

Received: February 17, 2020; Revised: June 5, 2020; Accepted: June 5, 2020; Available online: July 7, 2020

https://doi.org/10.5507/bp.2020.025

(c) 2021 The Authors; https://creativecommons.org/licenses/by/4.0/

${ }^{a}$ Clinic of Pediatrics, 1 st Faculty of Medicine, Charles University in Prague and Institute of Postgraduate Medical Education, Thomayer Hospital, Prague 4, Czech Republic

${ }^{b}$ National Reference Centre for Epidemiological Data Analysis, Prague 10, Czech Republic

'National Tuberculosis Surveillance Unit, Bulovka Hospital, Prague 8, Czech Republic

${ }^{d}$ Department of Epidemiology,2nd Faculty of Medicine, Charles University in Prague and University Hospital Motol, Prague 5, Czech Republic Corresponding author: Karolina Dolezalova, e-mail: karolina.dolezalova@ftn.cz

\section{INTRODUCTION}

From the global point of view, tuberculosis is the tenth highest cause of death, rising to fourth where it concerns causes of death due to infection. Per year, tuberculosis afflicts around 10 million people, of which 1.2 million $\operatorname{die}^{1}$. The epidemiology situation in the Czech Republic regarding the incidence of tuberculosis is significantly better. The incidence has been decreasing over the long-term and is the lowest among neighboring EU countries ${ }^{2,3}$. The mortality rate is also decreasing ${ }^{4}$.

This favorable trend was not interrupted by the ending of widespread calmetization in 2010, after which there was just a slight increase in the number of tuberculosis cases among the youngest age group (0-5 years). However, in contrast, the incidence of infections caused by nontuberculous mycobacteria continues to increase. Among children, this is well illustrated by the increase in occurrence of cervical lymphadenitis caused by Mycobacterium avium complex over the past 8 years.

Nontuberculous mycobacterial infections (NTM) are conditions caused by nontuberculous mycobacteria. These are ubiquitous saprophytes. To date, 200 species have been identified, of which 120 are pathogenic for humans. Nontuberculous mycobacteria reservoirs are fresh water, salt water, soil and biofilms. So far, no human-to-human transmission has been reported.

The most common species of mycobacteria are the MAC - Mycobacterium avium complex (which includes $M$. avium and $M$. intracellulare), $M$. kansasii, $M$. xenopii 200 (corrected according to reviewer N. 2) M. gordonae, M. chelonae, M.abscessus, M. scrofulaceum, M. malmoense and M. ulcerans.

The most common mechanism for the transmission of NTM infections is the inhalation of aerosol from natural or artificial reservoirs, i.e. from infected water reservoirs, terminal outlets of water pipelines in industrial establishments or households ${ }^{5,6}$.

In children, unilateral cervical lymphadenitis is typical. It is an illness affecting children up to the age of 5 years, with slight predominance in girls. The infectious agent predominantly enters through the orofacial area through the consumption of infected water or food. The predisposed group includes teething children. The invasion of mycobacteria into the blood stream first manifests as a regular infection of the upper respiratory tract and 
reactive adjacent lymph nodes. The swelling gradually increases. There is a granulomatous inflammation in the nodes and colliquation may occur. The inflammation spreads to the surrounding tissue, skin and subcutaneous tissue and typically manifests as unilateral swelling or swelling of the entire lymp node packet, most commonly the submandibular. The condition typically lasts for several weeks to months. The disease is not accompanied by a significant inflammatory response that would show up in laboratory results; there is only an increase in the erythrocyte sedimentation rate. An ultrasound is used for the diagnostics. A typical ultrasound image shows lymph node packets with central colliquation and sometimes calcification. In broader findings, the imaging may be extended to include magnetic resonance. A skin test with Mycobacterium avium sensitine is usually positive. To confirm the diagnosis, histological findings from extirpated nodes (granulomatous inflammation, palisade of Langhans cells, central necrosis) and microbiological identification of the causing microorganism using methods of molecular biology and classical culturing are usually conclusive ${ }^{7}$.

Vaccination against tuberculosis is performed using a live attenuated Bacillus Calmette-Guérin vaccine, which is also referred to as calmetization. It prevents the development of severe forms of post-primary tuberculosis and, due to the cross-reactive immune response, has a protective effect against infections caused by atypical mycobacteria. In post-war Czechoslovakia, widespread calmetization was introduced in 1953. This, in combination with public health and social programs against tuberculosis, led to a dramatic decrease in the incidence of tuberculosis by the beginning of the 21st century. The low incidence of tuberculosis in the Czech Republic, the relatively high reactogenicity of the vaccine and only the partial protective effect of the BCG vaccine against tuberculosis, despite quite common adverse effects, resulted in the eventual termination of the widespread mandatory vaccination of newborns, as was also the case in the majority of developed countries. Since November 1, 2010, only infants from risk groups are vaccinated on the basis of an assessment of a completed Tuberculosis Risk Questionnaire. The protective effect of the BCG vaccine against nontuberculous mycobacterial infections has been demonstrated on mouse models ${ }^{8}$. The increase in incidence of lymphadenitis caused by nontuberculous mycobacteria in those countries that withdrew the widespread vaccination program has been repeatedly documented ${ }^{9-14}$.

\section{METHOD}

Searching for cases: For the purposes of this study, we used data from the Tuberculosis Register. The register has existed in the Czech Republic since the 1950's. The reporting of new cases of the disease, relapses of tuberculosis and mycobacteriosis, as well as instances of death caused by tuberculosis and mycobacteriosis is a legal obligation for all doctors. The data regarding all reported cases are held centrally and monitored. The
Tuberculosis Register not only replaces the local gathering and processing of data from forms, but also helps to develop and maintain an integrated, good quality, up to date and secure - including in terms of personal data protection - database on the incidence of TB in the Czech Republic $^{3}$. The Czech Republic is an exception when it comes to record keeping and the mandatory reporting of nontuberculous mycobacteriosis because the majority of other countries do not report cases of mycobacteriosis.

For the purposes of this study, we chose diagnoses reported in the Tuberculosis Register under illness code A31.1 (skin mycobacterial infection), A31.8 (other mycobacterial infections) and A31.9 (mycobacterial infection, unspecified) according to the 10th revision of the International Classification of Diseases (ICD). The reported cases were confirmed by culturing and/or by methods of molecular biology and showed typical histological findings. To count incidence, population data was acquired from the Czech Statistical Office.

The assessment of trends was based on a Poisson regression. For graphical representation we used locally weighted scatterplot smoothing (lowess). A Chi-squared test was applied to compare the percentages in individual categories. The Mann-Whitney test was applied to analyze the continuous variables. The results of the statistical tests that had $P$-values lower than 0.05 were considered statistically significant.

\section{RESULTS}

In this article, results from two comparable 8-year intervals, namely 2003-2010 and 2011-2018, as reported in the Tuberculosis Register, are presented for the whole of the Czech Republic. This concerns 7 cases during the first time interval and 89 cases in the second time interval.

The average incidence per year in the first interval was $0.04 / 100000$ children and in the second interval $0.53 / 100$ 000 children. During the second time interval, there was an increase from $0.14 / 100000$ children in 2011 to $1.40 / 100000$ children in 2018. Fig. 1 and 2 illustrate the evolution of this trend over time using the lowess method. While the incidence during the first time interval did not show any time trend $(P=0.885)$, the year 2010 marks a significant turning point, with growth during the second interval being highly statistically significant $(P<0.001)$. Fig. 4 shows, that the incidence trends for NTM infections and tuberculosis were, during the observed period and for the age group up to 19 years, divergent and with a statistically significant difference $(P<0.001)$. Of all the cases, 57 (59\%) concerned girls and 39 (41\%) boys. During the first time interval, the ratio of girls to boys was 1.33:1, whilst during the second time interval it was 1.47:1. Gender representation did not statistically significantly differ between the intervals $(P=0.901)$. Fig. 3 illustrates the situation in the regions during both observed time intervals. The first group did not show the typical age distribution, with cases both among younger and older children; the median age was three years and the average age 4.4 years. Out of the seven children in the first group, 


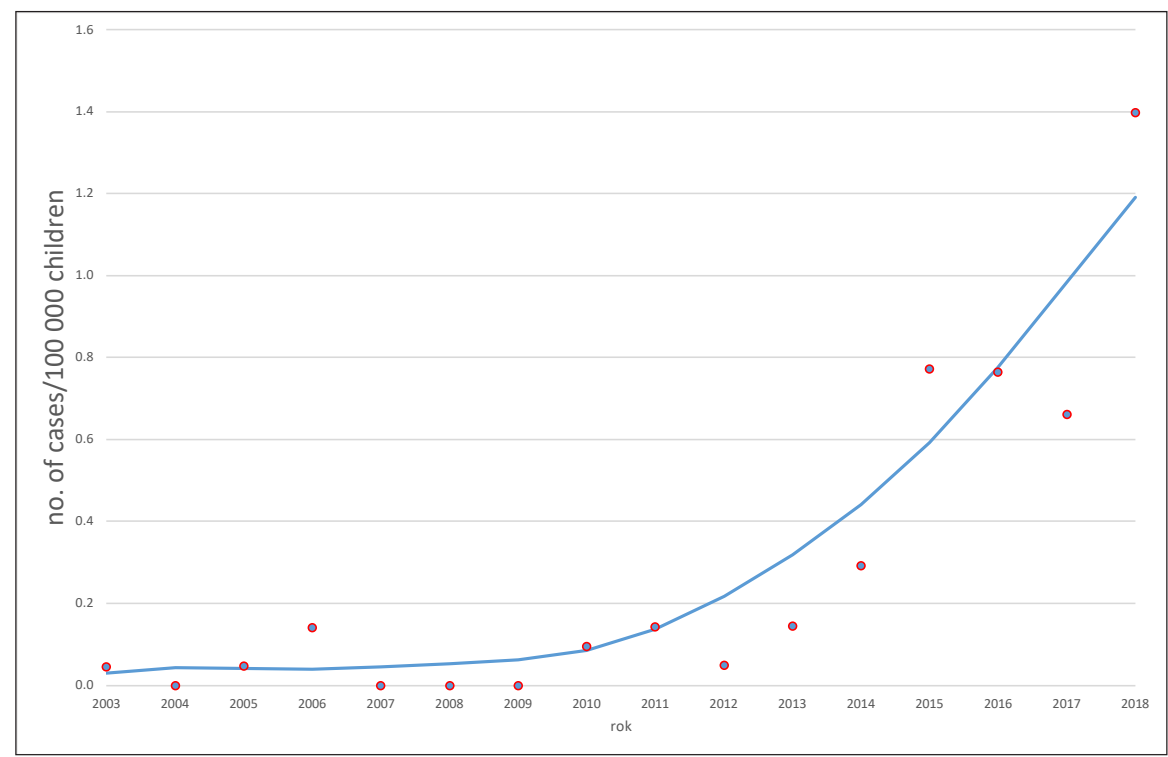

Fig. 1. Incidence of mycobacteriosis in the Czech Republic in the period 2003-2018.

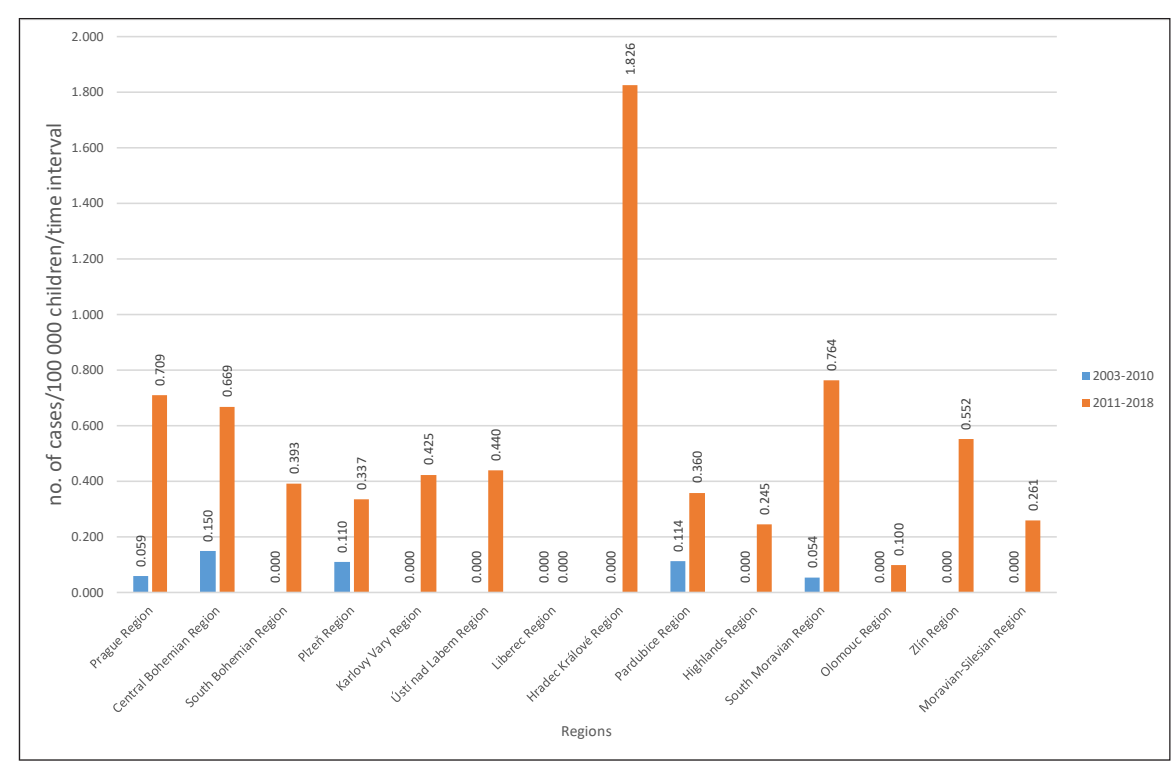

Fig. 2. Number of cases per 100000 children per time interval per region.

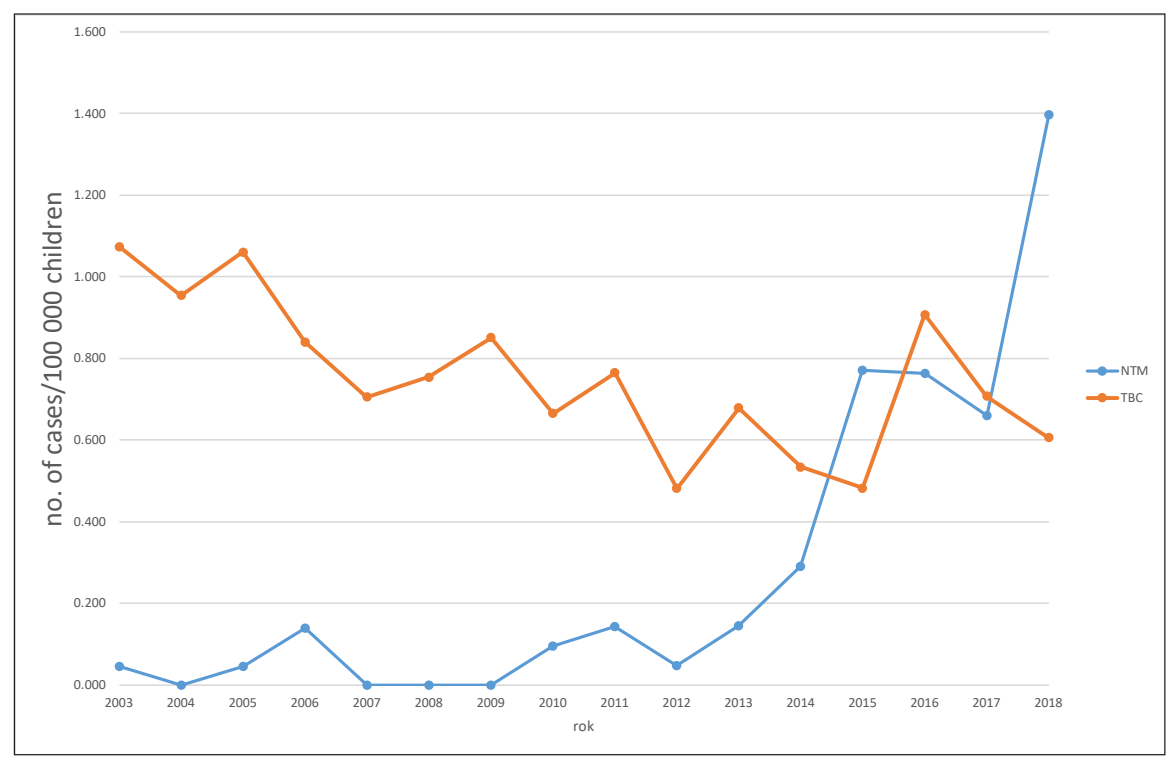

Fig. 3. Incidence of NTM and TB in the Czech Republic in the period 2003-2018. 
four were not vaccinated (unvaccinated children were an exception at the time of widespread mandatory vaccination) and three vaccinated. The second group consisted of only two vaccinated children, 82 not vaccinated children and five unconfirmed either way. Within the second observed group, the majority of cases (94\%) were in the age group 0-5 years, whereby the median age was two years and the average age 2.9 years. The cases were statistically significantly younger than in the first time interval $(P=0.012)$. (See attached graphs). In most cases, the cause was Mycobacterium avium (79\%), with one case caused by (1\%) Mycobacterium fortuitum; in $20 \%$ of the cases, mycobacteria were not specified or did not grow in the subcultures at all.

\section{DISCUSSION}

A comparison of the two time intervals showed that there was an indisputable increase in the incidence of cervical lymphadenitis caused by Mycobacterium avium complex in children and youngsters during the second time interval. The authors associate this trend with the termination of the widespread vaccination program against tuberculosis in 2010. In total, 89 described cases from the second time interval correspond with the expected characteristics, with a slight predominance in females and a median age of 2 years. This observation corresponds with studies on animals ${ }^{8}$ and previous clinical experiences and publications. Trnka et al. ${ }^{9}$ published data for the period 1986-1992 which revealed that when three regions in Czechoslovakia did not vaccinate, it led to an increase in the number of cases of tuberculosis and nontuberculous mycobacteriosis. In 2001, Vaníček et al. ${ }^{10}$ published work in which they compared two cohorts of children in East Bohemia. The first cohort comprised of 104881 children that had not been vaccinated against tuberculosis. The second cohort comprised of 14356 children that had been vaccinated. The conclusion was that the risk of nontuberculous mycobacteriosis contraction was, at the 95\% confidence level, statistically significantly higher in the cohort of children that were not vaccinated against tuberculosis. The Swedish authors Romanus et al. ${ }^{11}$ also assumed the protective effect of calmetization against localized infections caused by atypical mycobacteria. In the period 1969-1974, when Sweden supported the widespread calmetization of all newborns, the incidence of atypical mycobacteriosis in children under 5 years of age was $0.06 / 100000$. In the period 1975-1985, the incidence within this group grew to 26.8/100 000. Mycobacterium avium was the most commonly isolated pathogen. In contrast, in neighboring Finland, which supported widespread vaccination against tuberculosis until 2006, cervical lymphadenitis was almost unheard of with an incidence rate of $0.3 / 100000$ children $^{10}$. This observation led the authors to conclude that calmetization had a protective effect against NTM infections in children. The French authors Lacroix et al. ${ }^{13}$ presented the same conclusion, describing an increase of Mycobacterium avium bacteriosis in children under 5 years of age in France from 0.57/100
000 to $3.7 / 100000$ during an interval of 6 years after the ending of the country's vaccination program in 2007. The Dutch authors Haverkamp, et al. ${ }^{14}$ reviewed the results of a two-year national study, the results of which correspond with our observations. Their study revealed the following data: incidence rate of 77/100 000 children, median age 31 months, predominance in girls, $98.3 \%$ of the children were not vaccinated against tuberculosis. There are many more references and studies on the same subject, however, we do not consider it necessary to present more of them to illustrate the point any further.

Within the aforementioned context, it is important to ask whether the termination of widespread calmetization is the only factor that contributed to the described increase in infections caused by nontuberculous mycobacteria. A long-term low incidence of tuberculosis in developed countries, as evidenced by the referenced studies, may be considered another possible factor. A study by Orme and Collins performed on mice models ${ }^{8}$ confirmed that mice that suffered Mycobacterium tuberculosis infections subsequently had a more efficient immune response against nontuberculous mycobacteria due to specific memory T-lymphocytes. It is therefore probable that when the incidence rate of tuberculosis was higher, the immune system's protection against nontuberculous mycobacteriosis was better.

Likewise, immune disorders may be another contributory factor for the increase in the number of NTM infections. For example, the Mendelian Susceptibility to Mycobacterial Diseases is a group of monogenically conditioned primary immunodeficiencies caused by defects in the individual components of signaling pathway IL12-IL23/IFN $\gamma$, which ensures communication between phagocytes and Th1 lymphocytes. The disorders manifest themselves selectively in the form of decreased resistance to mycobacteria (especially against opportunistic pathogens) and non-typhoidal salmonellae, whilst the remaining antimicrobial immunity remains intact. The severity of the disease ranges from local complications at the place of inoculation with the BCG vaccine to fatal disseminated infection ${ }^{15}$. However, this group of diseases is very rare and there are only 5 patients currently diagnosed with it in the Czech Republic. Furthermore, most of the children from our cohort were immunocompetent. They were healthy children that were minimally ill before their nontuberculous mycobacterial infection, and also subsequently after surgery.

Other factors that need to be taken into consideration include the improvement of diagnostics. In recent years, genetic techniques - PCR NTM - are being used to diagnose the causative agent. These methods have a $90.9 \%$ sensitivity and a $98.4 \%$ specificity ${ }^{16}$ and yield results much faster than regular culturing, which can take up to 9 weeks. In the Czech Republic, such an examination is performed by a pathologist. They determine the diagnosis of nontuberculous mycobacteriosis based on clinical suspicion and histological findings that are typical for a specific inflammation. The diagnosis is subsequently confirmed by culturing. 


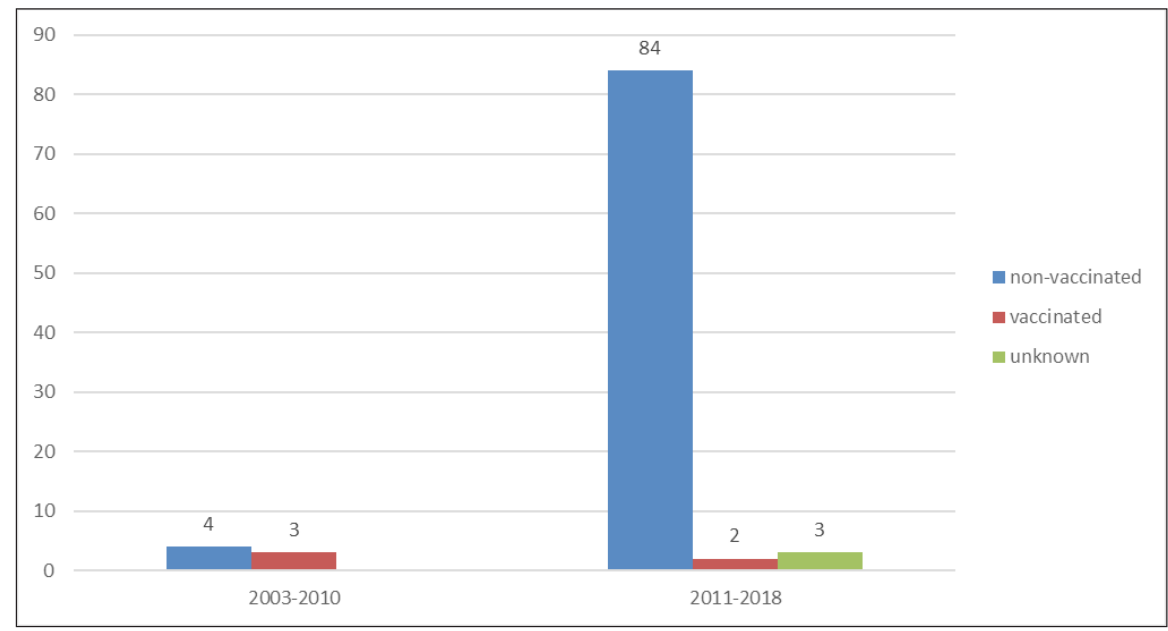

Fig. 4. Number of NMT cases according to the history of vaccination.

The results presented by the authors are based on mandatory reporting. These results may not be representative due to reporting bias. The infection is first reported by the doctor who determined the diagnosis and then by the laboratory that captured the positive culture test results. Despite this dual control system, there will undoubtedly be infections that escape the right diagnosis and, therefore, the correct reporting. Failure to perform correct mandatory reporting may be a contributory factor to the large variability among the individual regions. This reporting bias is likely the reason for the recorded interregional variability, representing both good and insufficient reporting policies, respectively.

The most common reason for under-reporting is the lack of sufficient diagnostics. If nontuberculous mycobacteria are not taken into consideration, they are not diagnosed during regular cultivation because they require a special culture medium and take several weeks to grow. Another reason for under-reporting is the human factor; the completion and dispatching of the mandatory form for reporting cases of tuberculosis and mycobacteriosis may be forgotten. For this reason, the authors assume that the actual number of cases is higher.

\section{CONCLUSION}

The indisputable increase in incidence of cervical lymphadenitis in the past eight years caused by nontuberculous mycobacteria, especially Mycobacterium avium, is probably linked to the ending of the widespread vaccination program against tuberculosis in 2010. This conclusion corresponds with the trend across many countries that have already withdrawn widespread $B C G$ vaccination programs.

This is in contrast to the incidence rate of tuberculosis in the Czech Republic, which shows a long-term downward trend. The withdrawal of widespread calmetization led to only a very minor increase in cases of tuberculosis in children, predominantly in the youngest age group (see Fig. 4).
Author contributions: KD: manuscript writing and literature search; DG: expert supervision, MM: statistical analysis, JW: consultant.

Conflict of interest statement: The authors state that there are no conflicts of interest regarding the publication of this article.

\section{REFERENCES}

1. Global tuberculosis report 2019. Geneva: World Health Organization; 2019. Licence: CC BY-NC-SA 3.0 IGO. Available from: https://www. who.int/tb/publications/global_report/en/ [cited 2020 Jan 6]

2. WHO Regional Office for Europe/European Centre for Disease Prevention and Control. Tuberculosis surveillance and monitoring in Europe 2019 - 2017 data. Copenhagen: WHO Regional Office for Europe; 2019. Available from: https://www.ecdc.europa.eu/ en/publications-data/tuberculosis-surveillance-and-monitoringeurope-2019 [cited 2020 Jan 6]

3. Basic review of the epidemiological situation in the incidence of tuberculosis in the Czech republic in $2018 \mathrm{http} / / /$ www.uzis.cz/katalog/ zdravotnicka-statistika/tuberkuloza-respiracni-nemoci, [cited 2020 Jan 6]

4. Doležalová K, Křepela K, Houšt'ková H. Incidence of tuberculosis and mycobacterial infections in the Czech republic in years 20002015. Cz-Sk Ped 2016;71(7-8):324-9. (In Czech)

5. Kubín M. A half century of nontuberculous mycobacterial infections in the Czech Republic and abroad. Studia Pneum et Phthis 2008;68(2):87-8. (In Czech)

6. Bártů V. Nontuberculous mycobacterioses. Postgrad Med 2008;10(6):604-7. (In Czech)

7. Školoudík L, Chrobok V, Ryšková L, Dědková J, Laco J, Vaníček H. Nontuberculous mycobacterial cervical lymphadenitis in childhood. Pediatr for Practise 2018;19(5):244-7. (In Czech)

8. Orme IM, Collins FM. Prophylactic effect in mice of BCG vaccination against nontuberculous mycobacterial infections. Tubercle 1985;66(2):117-20.

9. Trnka L, Daňková D, Švandová E. Six years' experience with the discontinuation of BCG vaccination. 4. Protective effect of BCG vaccination against the Mycobacterium avium intracellulare complex. Tuber Lung Dis 1994;75(5):348-52. doi: 10.1016/0962-8479(94)90080-9

10. Vaníček H, Pozler O. Incidence of mycobacterial infections in children. Postgraduate thesis. Prague 2001.

11. Romanus V, Hallander HO, Wåhlén $P$, Olinder-Nielsen $A M$, Magnusson $\mathrm{PH}$, Juhlin I. Atypical mycobacteria in extrapulmonary disease among children. Incidence in Sweden from 1969 to 1990, related to changing BCG-vaccination coverage. Tuber Lung Dis 1995;76(4):30010. doi: 10.1016/s0962-8479(05)80028-0 
12. Katila ML, Brander E, Backman A. Neonatal BCG vaccination and mycobacterial cervical adenitis in childhood. Tubercle.1987;68(4):291-6. doi: 10.1016/0041-3879(87)90070-5

13. Lacroix A, Piau C, Lanotte P, Carricajo A, Guillouzouic A, Peuchant O, Cady A, Dupin C, Fangous MS, Martin C, Cariou ME, Le Gall F, Bemer P, Tattevin P, MYCOMED Group. Emergence of Nontuberculous Mycobacterial Lymphadenitis in Children After the Discontinuation of Mandatory Bacillus Calmette and Guerin Immunization in France. Pediatr Infect Dis J 2018;37(10):257-60. doi: 10.1097/ INF.0000000000001977
14. Haverkamp MH, Arend SM, Lindeboom JA, Hartwig NG, van Dissel JT. Nontuberculous mycobacterial infection in children: a 2-year prospective surveillance study in the Netherlands. Clin Infect Dis 2004;39(4):450-6. doi: 10.1086/422319

15. Bloomfield $M$, Havránková $E$, Houšt'ková $H$, Kabíček $P$, Křepela $K$, Šedivá A, Bustamante J. Mendelian Susceptibility to Mycobacterial Diseases. Cz-Sk Ped 2016;71(7-8):340-4. (In Czech)

16. Perng CL, Chen HY, Chiueh TS, Wang WY, Huang CT, Sun JR. Identification of non-tuberculous mycobacteria by real-time PCR coupled with a high-resolution melting system. J Med Microbiol 2012;61(7):944-51. doi: 10.1099/jmm.0.042424-0 